\title{
Analysis of radiation patterns of rectangular microstrip antennas with uniform substrate
}

\author{
A. Boualleg, N. Merabtine \\ Laboratory LET Electronics Department Faculty of Engineering \\ University of Constantine (Algeria) \\ bouadzdz@yahoo.fr,na_merabtine@hotmail.com
}

\begin{abstract}
Microstrip antennas are useful as antennas mounted on moving vehicles such as cars, planes, rockets, or satellites, because of their small size, light weight and low profile. Since its introduction in 1985, the features offered by this antenna element have proved to be useful in a wide variety of applications, and the versatility and flexibility of the basic design have led to an extensive amount of development and design variations by workers hroughout the world.
\end{abstract}

Keywords: rectangular microstrip antennas, dielectric substrate, resonant microstrip, radiation patterns.

Manuscript received 03.07.05; accepted for publication 25.10.05.

\section{Introduction}

Microstrip antennas have been the subject to study for many years. Their analyses include the transmission line model [1], the cavity model [2], and the method of moments [3]. The physical size of a microstrip antenna is small, but the electrical size measured in wavelength $\lambda$ is not so small. Much research has gone into further reducing the microstrip antenna physical size.

Rectangular microstrip antennas have received much attention due to their major advantage of conformability.

In this paper, we consider only rectangular patches and discuss the aperture models for calculating the radiation patterns of the antenna using the Fourier integrals. The resonance problem has also been studied. However, the excitation problem was not treated.

\section{Analysis}

Fig. 1 shows a rectangular microstrip antenna fed by a microstrip line. It can also be fed by a coaxial line, with its inner and outer conductors connected to the patch and ground plane, respectively.

The height $h$ of the substrate is typically of a fraction of the wavelength, such as $h=0.05 \lambda$, and the length $L$ is of the order of $0.5 \lambda$. The structure radiates from the fringing fields that are exposed above the substrate at the edges of the patch.

In the so-called cavity model, the patch acts as resonant cavity with an electric field perpendicular to the patch, that is, along the Z-direction. The magnetic field has vanishing tangential components at the four edges of the patch. The fields of the lowest resonant mode (assuming $L \geq W$ ) are given by:

$$
\begin{array}{ll}
E_{z}(x)=-E_{0} \sin \left(\frac{\pi x}{L}\right) & \text { for }-\frac{L}{2} \leq x \leq \frac{L}{2}, \\
H_{y}(x)=-H_{0} \cos \left(\frac{\pi x}{L}\right) & \text { for }-\frac{W}{2} \leq y \leq \frac{W}{2},
\end{array}
$$

where $H_{0}=-j E_{0} / \eta$. We have placed the origin at the middle of the patch (note that $E_{z}(x)$ is equivalent to $E_{0} \cos (\pi x / L)$ for $\left.0 \leq x \leq L\right)$.

It can be verified that Eq. (1) satisfy Maxwell's equations and the boundary conditions, that is, $H_{y}(x)=0$ at $x= \pm L / 2$, provided the resonant frequency is $[4,5]$ :

$$
\omega=\frac{\pi c}{L} \Rightarrow f=0.5 \frac{c}{L}=0.5 \frac{c_{0}}{L \sqrt{\varepsilon_{r}}} .
$$

Where $c=c_{0} / \sqrt{\varepsilon_{r}}, \eta=\eta_{0} / \sqrt{\varepsilon_{r}}$, and $\varepsilon_{r}$ is the relative permittivity of the dielectric substrate. It follows that the resonant microstrip length will be halfwavelength:

$$
L=0.5 \frac{\lambda}{\sqrt{\varepsilon_{r}}} .
$$

Fig. 2 shows two models for calculating the radiation patterns of the microstrip antenna. The model on the left assumes that the fringing fields extend over a small 

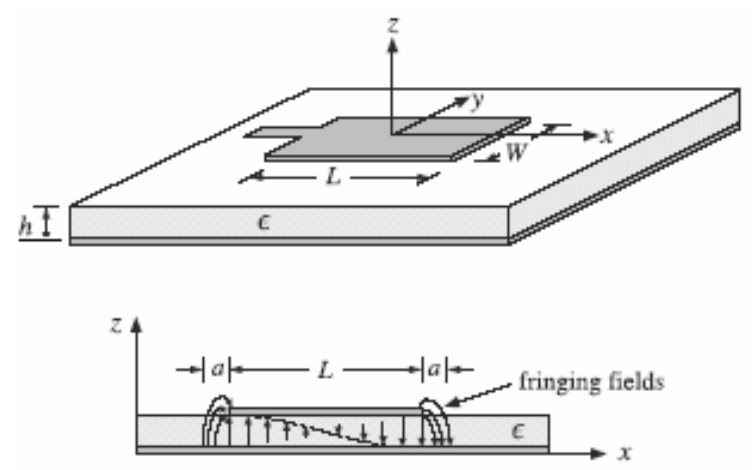

Fig. 1. Microstrip antenna and $E$-field pattern in substrate.

distance around the patch sides and can be replaced with the fields $E_{a}$ that are tangential to the substrate surface [6]. The four extended edge areas around the patch serve as the effective radiating apertures.

The model on the right assumes that the substrate is truncated beyond the extent of the patch [1]. The four dielectric substrate walls serve now as the radiating apertures.

The only tangential aperture field on these walls is $E_{a}=\hat{z} E_{z}$, because the tangential magnetic fields vanish by the boundary conditions.

For both models, the ground plane can be eliminated using the image theory, resulting in doubling the aperture magnetic currents, that is, $J_{m s}=-2 \hat{n} \times E_{a}$. The radiation patterns are then determined from $J_{m s}$.

For the first model, the effective tangential fields can be expressed in terms of the field $E_{Z}$ by the relationship: $a E_{a}=h E_{z}$. This follows by requiring the vanishing of the line integrals of $E$ around the loops labeled ABCD in the lower left of Fig. 2. Because $E_{z}= \pm E_{0}$ at $x= \pm L / 2$, we obtain from the left and right such contours:

$$
\begin{aligned}
& \int_{A B C D} E d l=-E_{0} h+E_{a} a=0, \\
& \int_{A B C D} E d l=E_{0} h-E_{a} a=0 \Rightarrow E_{a}=\frac{h E_{0}}{a} .
\end{aligned}
$$

In obtaining these, we assumed that the electric field is nonzero only along the sides $\mathrm{AD}$ and $\mathrm{AB}$. A similar argument for the sides 2 and 4 shows that $E_{a}= \pm h E_{z}(x) / a$. The directions of $E_{a}$ at the four sides are as shown in the figure. Thus, we have:

for sides 1 and $3: E_{a}=\hat{x} \frac{h E_{0}}{a}$.

for sides 2 and 4 :

$$
E_{a}= \pm \hat{y} \frac{h E_{z}(x)}{a}=\mp \hat{y} \frac{h E_{0}}{a} \sin \left(\frac{\pi x}{L}\right) .
$$

The outward normal to the aperture plane is $\hat{n}=\hat{z}$ for all four sides. Therefore, the surface magnetic currents $J_{m s}=-2 \hat{n} \times E_{a}$ become:

for sides 1 and 3: $J_{m s}=\hat{y} \frac{2 h E_{0}}{a}$,

for sides 2 and 4: $J_{m s}= \pm \hat{x} \frac{2 h E_{0}}{a} \sin \left(\frac{\pi x}{L}\right)$.

\section{Radiation fields}

The radiated electric field is obtained by [7]

$$
\begin{aligned}
& E=-j k \frac{e^{-j k r}}{4 \pi r} \hat{r} \times\left[\eta F \times \hat{r}-F_{m}\right], \\
& H=-\frac{j k}{\eta} \frac{e^{-j k r}}{4 \pi r} \hat{r} \times\left[\eta F+F_{m} \times \hat{r}\right]
\end{aligned}
$$

by setting $F=0$ and calculating $F_{m}$ as the sum of the magnetic radiation vectors over the four effective apertures:

$$
\begin{aligned}
& E=j k \frac{e^{-j k r}}{4 \pi r} \hat{r} \times F_{m}= \\
& =j k \frac{e^{-j k r}}{4 \pi r} \hat{r} \times\left[F_{m 1}+F_{m 2}+F_{m 3}+F_{m 4}\right] .
\end{aligned}
$$

The vectors $F_{m}$ are the two-dimensional Fourier transforms over the apertures:
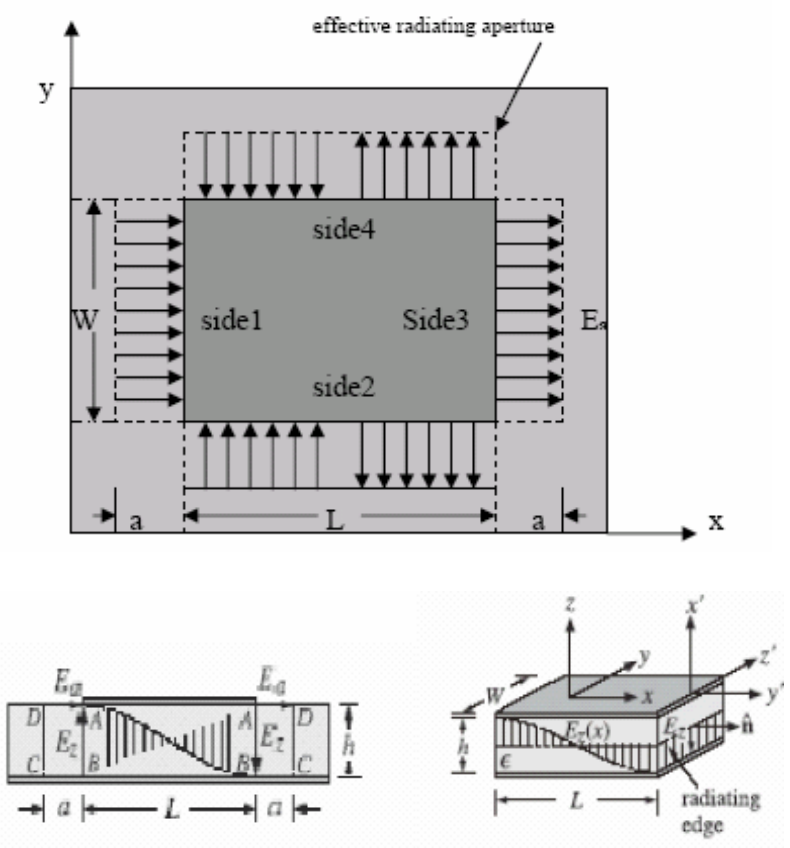

$-b-$

Fig. 2. Aperture models for microstrip antenna. 
The integration surfaces $d S=d x d y$ are approximately, $d S=a d y$ for 1 and 3 , and $d S=a d x$ for 2 and 4. Similarly, in the phase factor $e^{j k_{x} x+j k_{y} y}$, we must set $x=\mp L / 2$ for sides 1 and 3, and $y=\mp W / 2$ for sides 2 and 4. Inserting Eq. (5) into the Fourier integrals and combining the terms for apertures 1 and 3 as well as 2 and 4, we obtain:

$F_{m, 13}=\hat{y} \frac{2 h E_{0}}{a} \times$

$\times \int_{-W / 2}^{W / 2}\left(e^{-j k_{x} L / 2}+e^{j k_{x} L / 2}\right) e^{j k_{y} y} a d y$,

$F_{m, 24}=\hat{x} \frac{2 h E_{0}}{a} \times$

$\times \int_{-L / 2}^{L / 2}\left(e^{-j k_{y} W / 2}-e^{j k_{y} W / 2}\right) \sin \left(\frac{\pi x}{L}\right) e^{j k_{x} x} a d x$.

Using Euler's formulae and the integrals:

$$
\begin{aligned}
& \int_{-W / 2}^{W / 2} e^{j k_{y} y} d y=W \frac{\sin \left(k_{y} W / 2\right)}{k_{y} W / 2}, \\
& \int_{-L / 2}^{L / 2} \sin \left(\frac{\pi x}{L}\right) e^{j k_{x} x} d x=\frac{2 j k_{x} L^{2}}{\pi^{2}} \frac{\cos \left(k_{x} L / 2\right)}{1-\left(\frac{k_{x} L}{\pi}\right)^{2}},
\end{aligned}
$$

we find the radiation vectors:

$$
\begin{aligned}
& F_{m, 13}=\hat{y} 4 E_{0} h W \cos \left(\pi v_{x}\right) \frac{\sin \left(\pi v_{y}\right)}{\pi v_{y}}, \\
& F_{m, 24}=\hat{x} 4 E_{0} h L \frac{4 v_{x} \cos \left(\pi v_{x}\right)}{\pi\left(1-4 v_{x}^{2}\right)} \sin \left(\pi v_{y}\right),
\end{aligned}
$$

where we defined the normalized wavenumbers as usual:

$v_{x}=\frac{k_{x} L}{2 \pi}=\frac{L}{\lambda} \sin \theta \cos \phi$,

$v_{y}=\frac{k_{y} W}{2 \pi}=\frac{W}{\lambda} \sin \theta \sin \phi$.

Using some trigonometric identities, we may write the radiated fields from sides 1 and 3 in the form:

$$
\begin{aligned}
& E(\theta, \phi)= \\
& =j k \frac{e^{-j k r}}{4 \pi r} 4 E_{0} h W[\hat{\phi} \cos \theta \sin \phi-\hat{\theta} \cos \phi] F_{1,3}(\theta, \phi),
\end{aligned}
$$

where we defined the function:

$$
F_{1,3}(\theta, \phi)=\cos \left(\pi v_{x}\right) \frac{\sin \left(\pi v_{y}\right)}{\pi v_{y}} .
$$

Similarly, we have for sides 2 and 4 :

$$
\begin{aligned}
& E(\theta, \phi)= \\
& =j k \frac{e^{-j k r}}{4 \pi r} 4 E_{0} h L[\hat{\phi} \cos \theta \cos \phi+\hat{\theta} \sin \phi] F_{2,4}(\theta, \phi), \\
& F_{2,4}(\theta, \phi)=\frac{4 v_{x} \cos \left(\pi v_{x}\right)}{\pi\left(1-4 v_{x}^{2}\right)} \sin \left(\pi v_{y}\right)
\end{aligned}
$$

\section{Radiation patterns}

The normalized gain is found from Eq. (10) to be:

$$
\begin{aligned}
& g_{1,3}(\theta, \phi)=\frac{|E(\theta, \phi)|^{2}}{|E(\theta, \phi)|_{\max }^{2}}= \\
& =\left(\cos ^{2} \theta \sin ^{2} \phi+\cos ^{2} \phi\right)\left|F_{1,3}(\theta, \phi)\right|^{2} .
\end{aligned}
$$

The corresponding expression for sides 2 and 4, although not normalized, provides a measure for the gain in that case:

$$
g_{2,4}(\theta, \phi)=\left(\cos ^{2} \theta \cos ^{2} \phi+\sin ^{2} \phi\right)\left|F_{2,4}(\theta, \phi)\right|^{2} \text {. }
$$

The $E$ - and $H$-plane gains are obtained by setting $\phi=0^{\circ}$ and $\phi=90^{\circ}$ in Eq. (13):

$$
\begin{array}{ll}
g_{1,3_{E}}(\theta)=\left|\cos \left(\pi v_{x}\right)\right|^{2}, & v_{x}=\frac{L}{\lambda} \sin \theta, \\
g_{1,3_{H}}(\theta)=\left|\cos \theta \frac{\sin \left(\pi v_{y}\right)}{\pi v_{y}}\right|, & v_{y}=\frac{W}{\lambda} \sin \theta .
\end{array}
$$

Most of the radiation from the microstrip arises from sides 1 and 3. Indeed, $F_{1,3}(\theta, \phi)$ has a maximum towards broadside, $v_{x}=v_{y}=0$, whereas $F_{2,4}(\theta, \phi)$ vanishes. Moreover, $F_{2,4}(\theta, \phi) *$ for all $\theta$ and $\phi=0$ (E-plane) or $\phi=90^{\circ}$ (H-plane).

Therefore, sides 2 and 4 contribute little to the total radiation, and they are usually ignored.

\section{Numerical results and discussion}

Fig. 3 shows the $E$ - and $H$-plane patterns for $W=L=0.3356 \lambda$. Both patterns are fairly broad.

The choice for $L$ comes from the resonant condition $L=0.5 \lambda / \sqrt{\varepsilon_{r}}$. For a typical substrate with $\varepsilon_{r}=2.22$, we find $L=0.5 \lambda / \sqrt{2.22}=0.3356 \lambda$.

Fig. 4 shows the 3 -dimensional gains computed from Eqs (13) and (14). The field strengths (square roots of the gains) are plotted to improve the visibility of the graphs.

The gain from sides 2 and 4 vanishes along the $v_{x}$ and $v_{y}$ axes, while its maximum in all directions is $\sqrt{g}=0.1475$ or $-16.6242 d B$. 

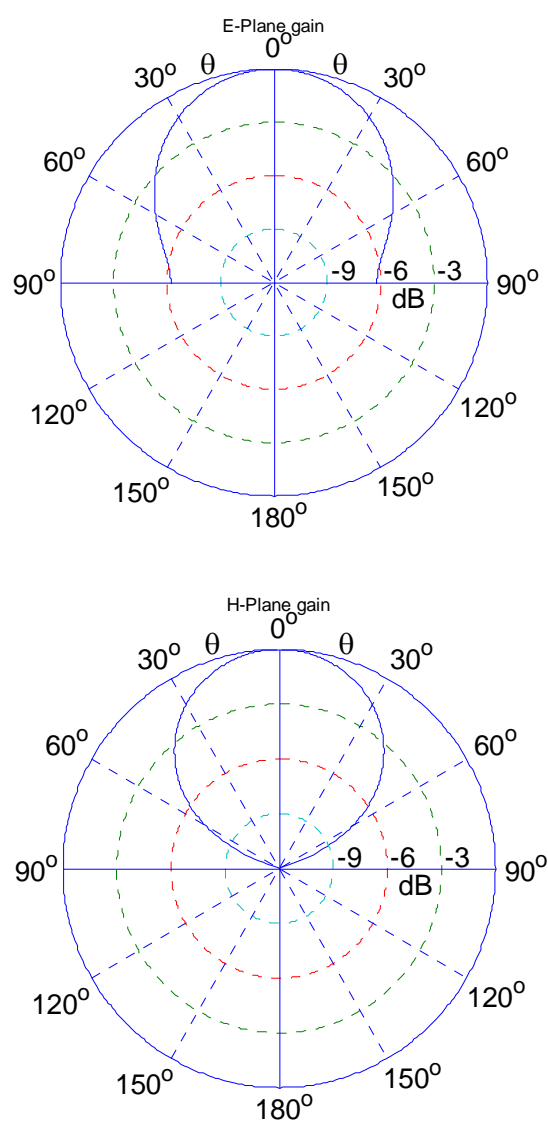

Fig. 3. $E$ - and $H$-plane gains of microstrip antenna.

\section{Conclusion}

Our study based on the analysis of radiation patterns of the left model of Fig. 2. It found the most of the radiation from the microstrip arises from sides 1 and 3 . On the other hand, the radiation from sides 2 and 4 vanishes almost completely.

Using the alternative aperture model shown on the right of Fig. 2, one obtains identical expressions for the magnetic current densities $J_{m s}$ along the four sides, and therefore, identical radiation patterns. The integration surfaces are now $d S=h d y$ for sides 1 and 3 , and $d S=h d x$ for 2 and 4.

\section{References}

1. A.G. Demeryd, Linearly polarized microstrip antennas // IEEE Trans. Antennas Propagat. AP-24, p. 846-851 (1976).
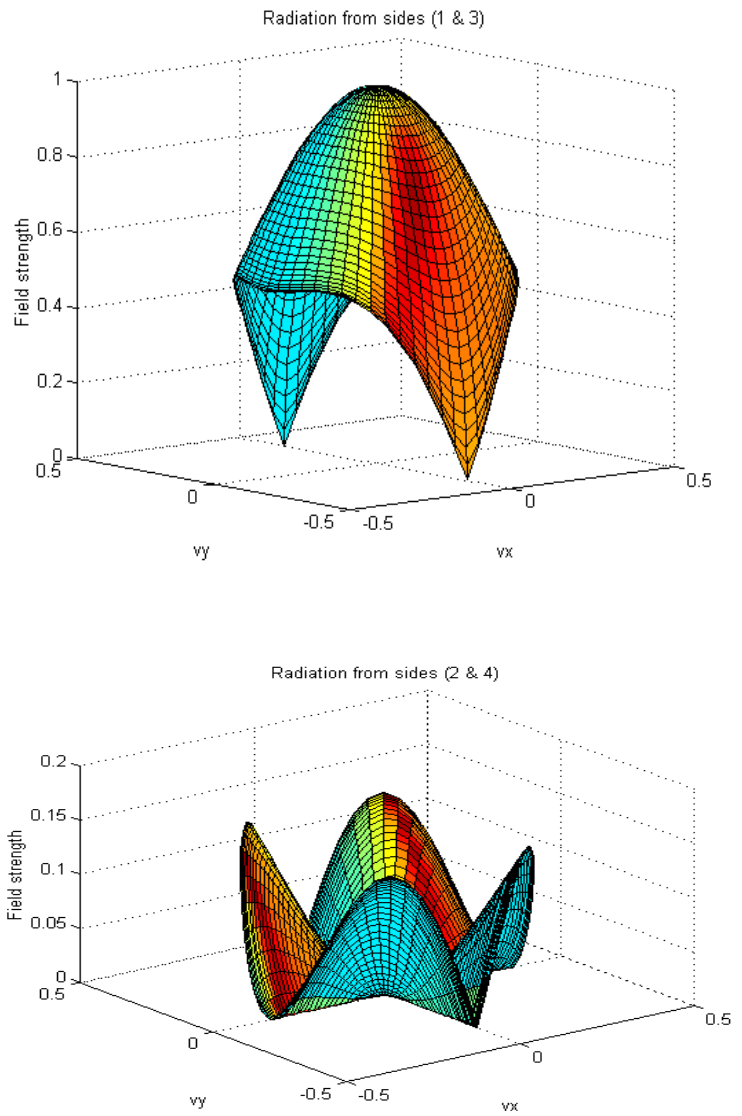

Fig. 4. Three-dimensional gain patterns from sides 1 and 3 as well as 2 and 4 .

2. Y.T. Lo, D. Solomon, and W.F. Richards, Theory and experiment on microstrip antennas // IEEE Trans. Antennas Propagat. AP-27, p. 137-145 (1979).

3. E.H. Newman and P. Tulyathan, Analysis of microstrip antennas using moment methods // IEEE Trans. Antennas Propagat. AP-29, p. 47-53 (1981).

4. S.M. Mi, T.M. Habashy, J.F. Kiang, and J.A. Kong, Resonance in cylindrical-rectangular and wraparound microstrip structures // IEEE Trans. Microwave Theory Tech. 37, p. 1773-1789 (1989).

5. K.L. Won, Y.T. Cheng, and J.S. Row, Resonance in a superstrate-loaded cylindrical-rectangular microstrip structure // IEEE Trans.Microwave Theory Tech. 41, p. 814-819 (1993).

6. P. Hammer, D. Van Bouchaute, D. Verschraeven, and A. Van de Capelle, A model for calculating the radiation field of microstrip antennas // IEEE Trans. Antennas Propagat. AP-27, p. 267 (1979).

7. C.A. Balanis, Antenna theory - analysis and design, John Wiley \& Sons, 2-ed., New York (1997). 